Document downloaded from:

http://hdl.handle.net/10251/169027

This paper must be cited as:

Alemany Díaz, MDM.; Alarcón Valero, F.; Pérez Perales, D.; Guyon, C. (2020). A Decision Support Tool for the Selection of Promoting Actions to Encourage Collaboration in Projects for the Agriculture Sector. IFIP Advances in Information and Communication Technology. 598:534-545. https://doi.org/10.1007/978-3-030-62412-5_44

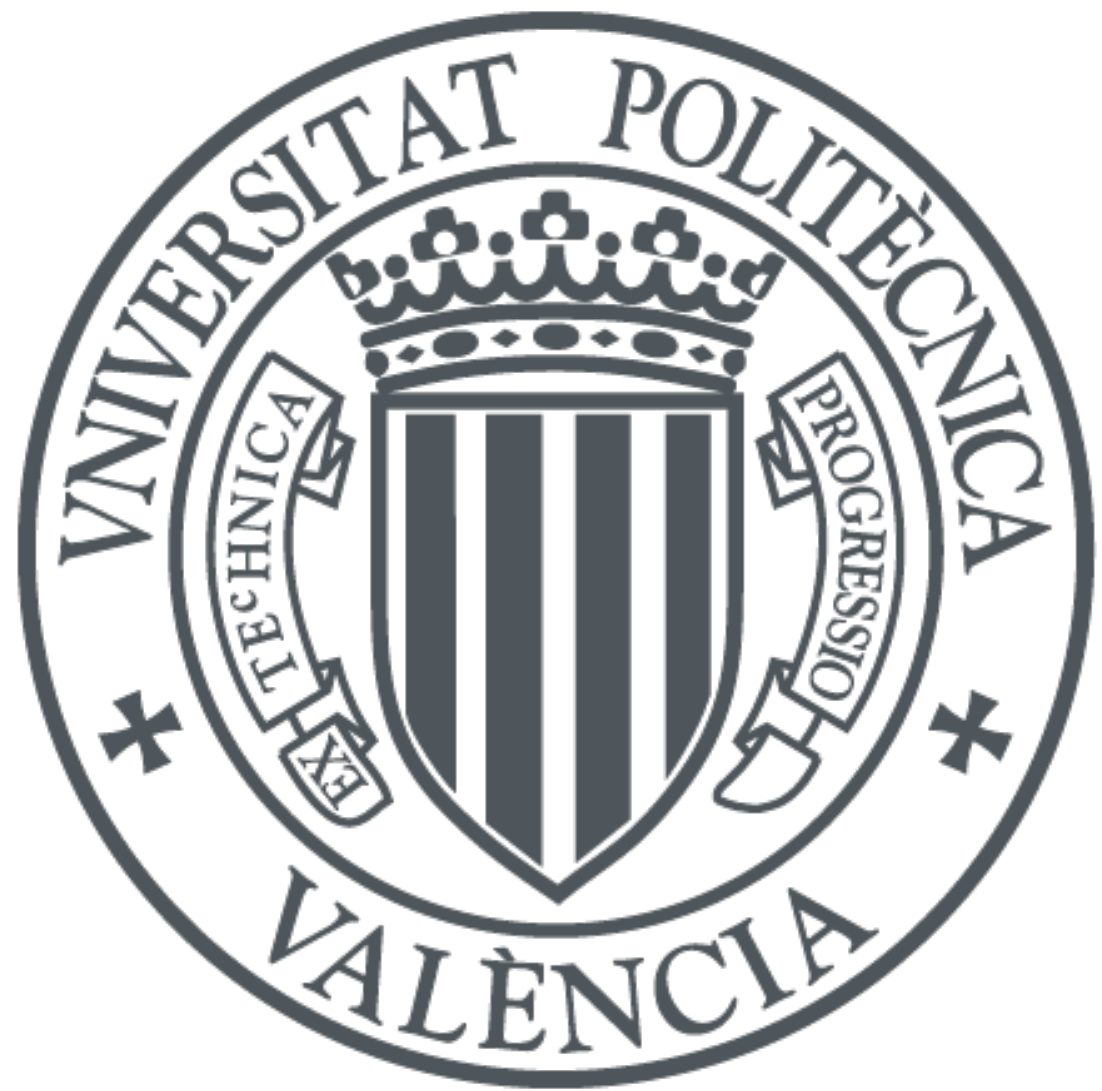

The final publication is available at

https://doi.org/10.1007/978-3-030-62412-5_44

Copyright Springer

Additional Information 


\title{
A Decision Support Tool for the Selection of Promoting Actions to Encourage Collaboration in Projects for the Agriculture Sector
}

\author{
MME Alemany ${ }^{1}$, F Alarcón ${ }^{1}$, D Pérez ${ }^{1}$, C Guyon $^{2}$ \\ ${ }^{1}$ Research Centre on Production Management and Engineering (CIGIP). Universitat \\ Politècnica de València. Camino de Vera S/N, 46022 València, Spain; ${ }^{2}$ Bretagne Development \\ Innovation. 1bis Route de Fougères, 35510 Cesson-Sévigne, France \\ \{mareva, faualva, dapep\}@cigip.uvp.es, c.guyon@bdi.fr
}

\begin{abstract}
Development and innovation agencies promote consortiums of agricultural stakeholders to collaborate in the proposal of projects for public calls. To achieve this partnerships, these agencies should select between different promoting actions to be performed with two objectives: maximize the number of project proposals presented and minimize the resources invested. To support agencies with these decisions, a computer tool based on a multiobjective integer linear programming model is proposed. To deal with the two objectives the weighting sum method is implemented. The model is validated in different scenarios by means a realistic case of an agency in Brittany (France). The results show the conflict between the two objectives considered and the dependency of the solutions on the scenarios defined. As a conclusion it can be stated that: 1) decision-makers should be careful in defining the weights of each objective and 2) the impact of the different promoting actions on the level of stakeholders' participation should be precisely estimated.
\end{abstract}

Keywords: Decision Support, Optimization, Collaboration, Project Proposals, Agriculture

\section{Introduction}

Many EU-funded research and innovation programmes have been developed since 1990, such as INTERREG, H2020, Intelligent Energy Europe, LIFE, etc. Others, such as Europe-Horizon (2021-27) are coming soon. Not only EU-funded but also a high number of national and regional programmes have been launched in the last three decades. These programmes aim to drive economic growth and create jobs. They do special emphasis on excellent science, industrial leadership and tackling societal challenges. The goal is to ensure Europe produces world-class science, removes barriers to innovation and makes it easier for the public and private sectors to work together in delivering innovation and collaborative work.

At the operational side, these EU, national or regional programmes may comprise one or more thematic sections, which, in turn, describe their overall objectives, the 
respective calls for proposals, and the topics within each call. Besides, some general annexes describing general rules such as standard admissibility conditions and eligibility criteria, selection and award criteria are presented [1].

One of the problems that some entities face is deciding on which topic or topics from a certain call to make project proposals, since a multitude of them can be presented in each topic. Besides, these entities are often engaged in several programmes, what increases the number of calls and makes the decision-making process more complex due to the limited resources. These entities must assess then what are the inputs (resources, costs...) and outputs (benefits) of presenting projects proposals in different topics of each program call.

Among other data, they must account for the planned start and end dates of the projects (in case they are approved), some strict requirements about the number and type of partners that can participate as well as their objectives or budget.

Finally, these entities, taking into consideration all the previous issues, decide to present a certain number of project proposals, of which only some of them will be approved. These approved proposals will become firm projects and be developed within the defined terms and conditions.

The described problem may be included within what is well known in the literature as project selection problem, considered as the first essential part of project portfolio management. It consists in selecting from a large set of projects, a subset of projects to be undertaken [2].

A large amount of methods for project selection are presented in the literature, basically divided into two categories.

First ones, are based on the multi-objective nature (basically return and risk) of the problem [3]. Different techniques (quantitative and qualitative) to select projects according to these multi-objective criteria are proposed in deterministic and uncertain environments. Regarding deterministic techniques, three are considered as the most popular: analytic hierarchy process (AHP), analytic network process (ANP) and order of preference by similarity to ideal solution (TOPSIS). In stochastic scenarios researchers usually consider uncertainty in the form of fuzzy or interval data and proposed more sophisticated method for ranking projects.

Second ones are based on optimization models, based on operation research tools. Some form of mathematical programming is used to select a set of projects which deliver the maximum benefit represented by an objective function subjected to a series of constraints. They consider relationships between projects and other factors that first ones do not consider [4]. Among the major deterministic optimization models are linear and non-linear programming, integer algorithms, dynamic programming and goal programming [5]. The inclusion of the uncertainty is well described in [6], where a brief review of fuzzy mathematical programming and a comparison with stochastic programming in portfolio selection problem is conducted.

Nevertheless, most of the reviewed works only deal with R\&D projects conducted by companies from different sectors or industries to innovate and introduce new products and services or to improve their existing offerings. Typical sectors that invest the most are technological [7], construction [8], energy [9]-[10], etc.

Only a few works address the project selection problem as described at the beginning of this introduction, and if so, it is usually done from the perspective of the institution that launches the calls for projects [11]-[12], as they must select, once 
received the project proposals from the different entities, which projects to finance or co-finance with public or private aid.

The literature lacks work in which the project selection is made from the perspective of the agencies whose business is based on disseminating the data and interest of different calls and to encourage the creation of consortia among different partners for the development of projects proposals of which they previously know the selection procedure and criteria. The creation of consortia involves the choice of partners to carry out the projects in a collaborative network context. Selecting partner(s) for collaborative projects is the main challenge that organizations face before they can attain the advantages of collaboration [13]. Collaboration is defined in [14] as process in which entities share information, resources and responsibilities to jointly plan, implement, and evaluate a program of activities to achieve a common goal. In this task of boosting collaborative networks for projects development, even less, research has been done to support these agencies to decide about what types of actions to be done in order to promote project proposals of different partners.

Partially related to this, only a research stream that addresses the partners' selection problem have been found in the literature. [15] develop a conceptual model indicating how an organization should choose the right partners for a set of projects. [16] present an explorative empirical study that shows the steps in the partner selection process. [17] formulates a multicriteria best value source selection methodology for public-private partnerships projects. But these studies neither include optimization models based on mathematical programming nor with the characteristics addressed in the problem under study.

To fill the above gap, in this paper a multi-objective integer linear programming (ILP) model for project selection in this specific scenario is proposed. The main contribution of this paper is to address the project selection problem not from the perspective of the institution that launches the calls for projects but from the perspective of agencies boosting the creation of collaborative networks. These agencies should select the investment in promoting actions to establish consortia among supply chain stakeholders for project proposals. Additionally, the selection of stakeholders (partners) at each supply chain stage to create collaborative networks to participate in different projects proposals presented at each call should be made. In doing so, it is necessary to consider different constraints related to the potential participation of stakeholders in calls based on their previous knowledge and expertise, the maximum number of projects in which each stakeholder can simultaneously participate at each period and the available capacity of the resources to carry out the marketing actions by the agency. In doing so, besides the traditional objective of maximizing the number of projects proposals presented, the resources allocated by the agency to the promoting actions is aimed also to be minimized. These marketing actions are crucial to encourage different companies to be engaged in different partnerships and therefore presenting project proposals.

The rest of the paper is arranged as follows. Section 2 describes the problem being studied, as is the case of an agency that supports and encourages the implementation of projects of different nature. In Section 3, a deterministic ILP model to solve the problem is presented. Section 4 reports its application to a realistic case based on 
different scenarios that validate the model. Finally, some conclusions and future research lines are drawn in Section 5.

\section{Problem Description}

The configuration of the agricultural supply chain under study is assumed to be composed by three stages (see Fig. 1):

- The stage 1 corresponds to the experimental laboratories that test and evaluate the technology developed by the technology manufacturers.

- The stage 2 is integrated by manufacturers of the technology for the agricultural companies

- The stage 3 makes reference to the agricultural companies.

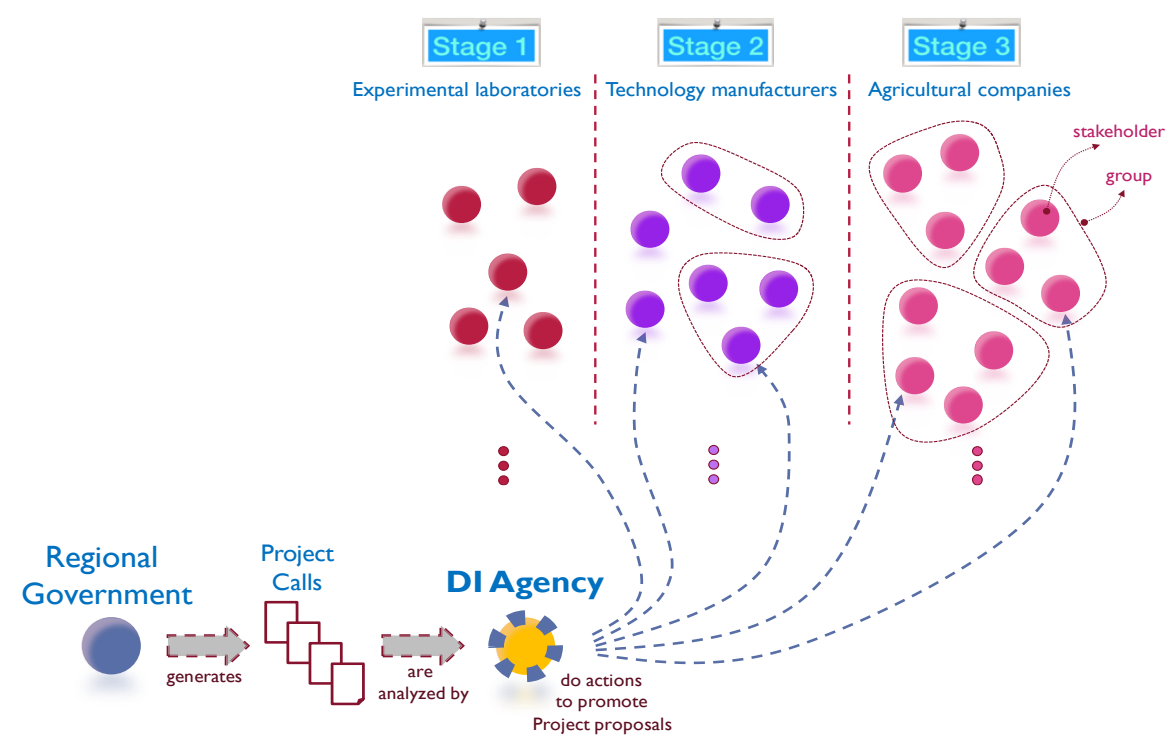

Fig. 1. Configuration of the agricultural supply chain.

In addition, there is an agency dedicated to promote development and innovation projects in the region. This agency, usually of governmental character, promotes collaboration among stakeholders of the different stages to encourage the consortia development to present as many project proposals as possible. These projects respond to regional calls that specify:

- A start and end date for the presentation of project proposals,

- A topic, which may generate more or less interest among stakeholders of different stages of the supply chain and fit in a lesser or greater extent the skills and previous experiences of the stakeholders,

- Economic endowment, which make the call for companies more or less attractive. 
Due to the budget limitations, for each call a limited number of projects can be presented. To ensure that a project proposal has some possibility of being accepted in a specific call a minimum number of stakeholders of the different stages is required. Besides, a maximum number is desirable in order to apply for several projects in the same call. Indeed, the consortia for each project can be made up of any number of companies at the third stage, combined or not with suppliers at the first or second stage. Nevertheless, if there are stakeholders at the first stage in the consortium, there may also be a variable number of stakeholders belonging to the second stage. Stakeholders of the same stage that are interested in the same project calls will integrate a group of interest. Because regional calls can be overlapped during different time periods along the year, it is assumed that each group of stakeholders can work simultaneously in the preparation of a limited number of project proposals.

In order to encourage the creation of projects, the agency dedicated to the innovation and development, designs actions, plans them, carries them out, evaluates the results of the actions according to the number of projects proposals presented and calculates the costs of these actions so that it is possible to know in which actions there is a greater probability of success in relation to costs. These actions are aimed at disseminating the data and interest of each regional call and at encouraging the creation of consortiums for the development of projects. If the actions succeed in arousing the interest of the stakeholders these are associated in one or several consortiums and propose one or several projects respectively. These actions include the simple sending of an e-mail (low consumption of resources) until the organization of conferences, stakeholder meeting days (high consumption of resources), etc. So the agency is interested in knowing the actions to be done during each period of the year in order to achieve the maximum number of project proposal presented while also taking into account the resources invested.

\section{Mathematical Model Formulation}

The notation of the integer programming model used to support the Agency decision about the number of action types to be performed on each group of stakeholders belonging to the different stages for each project call is presented in Tables 1 to 3 .

Table 1. Nomenclature: indexes and sets.

\begin{tabular}{|c|c|c|}
\hline Indexes & Sets & \\
\hline $\begin{array}{ll}s & \text { Stage of the agriculture supply chain } \\
g & \text { Group of stakeholders with the same interests }\end{array}$ & $G_{s}$ & Group of stakeholders $g$ in stage $s$ \\
\hline$j \quad$ Project call & $I_{g}$ & Projects calls $j$ of interest for a group \\
\hline $\begin{array}{ll}p & \text { Proposal of projects to be presented } \\
a & \text { Promotion action type of the agency }\end{array}$ & & \\
\hline $\begin{array}{ll}t & \text { Time period } \\
r & \text { Resource type of the agency }\end{array}$ & $P_{j}$ & $\begin{array}{l}\text { Group of projects } p \text { that can be } \\
\text { proposed in a project call } j\end{array}$ \\
\hline
\end{tabular}


Table 2. Nomenclature: parameters

\begin{tabular}{|c|c|}
\hline Parameters & \\
\hline$n s_{g}$ & Number of stakeholders belonging to group $g$ \\
\hline$n j_{g}$ & Number of projects in which the stakeholders of group $g$ can simultaneously work \\
\hline $\operatorname{nsmin}_{j s}$ & Minimum number of stakeholders of stage $s$ required to participate in a project call $j$ \\
\hline $\operatorname{nsmax}_{j s}$ & Maximum number of stakeholders of stage $s$ allowed to participate in a project call $j$ \\
\hline $\operatorname{npmax}_{j}$ & Maximum number of projects that can be presented for the project call $j$ \\
\hline$i a_{a g j}$ & $\begin{array}{l}\text { Impact of one action type a on a group of stakeholders } g \text { expressed as the percentage } \\
\text { of the number of stakeholders in the group } g \text { that will be interested in participating in } \\
\text { the project call } j \text {. }\end{array}$ \\
\hline cons $_{\text {rajt }}$ & $\begin{array}{l}\text { Consumption of resource type } r \text { of the agency in doing action type a for the project } \\
\text { call } j \text { in } t\end{array}$ \\
\hline $\operatorname{cap}_{r t}$ & Available capacity of resource $r$ during the time period $t$ \\
\hline$\delta_{j t}$ & $\begin{array}{l}\text { Parameter with a value of } 1 \text { if the time period for presenting proposals to project call } \\
j \text { include period } t \text { and } 0 \text { otherwise }\end{array}$ \\
\hline
\end{tabular}

Table 3. Nomenclature: decision variables

\begin{tabular}{ll}
\hline Decision Variables \\
\hline$N_{s g j p}$ & $\begin{array}{l}\text { Number of stakeholders in stage } s \text { of group } g \text { participating in project } p \text { of call } j \\
Y_{s j p}\end{array}$ \\
$Y P_{j p}$ & $\begin{array}{l}\text { Binary variable with a value of } 1 \text { if some stakeholder of stage } s \text { participate in project } \\
\text { Binary variable with a value of } 1 \text { if project } p \text { is presented in project call } \mathrm{j} \text { and } 0\end{array}$ \\
$Y A_{\text {asgj }}$ & $\begin{array}{l}\text { otherwise } \\
\text { Binary variable with a value of } 1 \text { if action type a is performed in the group of } \\
\text { stakeholders } g \text { of stage } s \text { for the project call } j \text { and } 0 \text { otherwise }\end{array}$ \\
$Y A T_{a}$ & Total number of type action $a$ carried out by the agency \\
\hline
\end{tabular}

Different objectives are pursued when assigning the number of each action type to the different projects. The first one aims to maximize the number of projects proposals presented for all the project calls triggered during the horizon (1). The second one, aims to minimize the quantity of resources invested in these actions (2).

$$
\begin{gathered}
\operatorname{Max}[Z 1]=\sum_{j} \sum_{p \in P_{j}} Y P_{j p} \\
\operatorname{Min}[Z 2]=\sum_{r} \sum_{a} \sum_{s} \sum_{g \in G_{s}} \sum_{j \in J_{g}} \sum_{t \in T_{j}} \text { cons }_{\text {rajt }} Y A_{\text {asgj }}
\end{gathered}
$$

Both objectives are combined by means of the weighting sum method. In order to scale these objectives, each one is divided by their maximum value. This ensures that they both move in the range $[0,1]$. The maximum value can be obtained by maximizing only one objective at a time. The weights $w 1$ and $w 2$ represent the importance provided by the decision-maker to each objective in such a way that the more important the objective, the greater the weight assigned. It is noteworthy that the following relationship always apply: $w 1+w 2=1$. 


$$
\operatorname{Max}[Z]=w 1 \frac{Z 1}{Z 1 \max }-w 2 \frac{Z 2}{Z 2 \max }
$$

The following constraints should be respected. Each project proposal $p$ of a certain call $j$ requires a minimum (4) and a maximum (5) number of stakeholders of each stage in case this project is finally presented.

$$
\begin{array}{ll}
\operatorname{nsmin}_{j s} \cdot Y P_{j p} \leq \sum_{g \in G_{s}} N_{s g j p} & \forall s, j, p \in P_{j} \\
\sum_{g \in G_{s}} N_{s g j p} \leq \operatorname{nsmax}_{j s} \cdot Y P_{j p} & \forall s, j, p \in P_{j}
\end{array}
$$

The total number of stakeholders of each group at each stage participating in a specific call $\mathrm{j}$ should be no higher than the expected impact on each group of stakeholders produced by the action carried out (6).

$$
\sum_{p \in P_{j}} N_{s g p j} \leq \sum_{a}\left(i a_{a g j} \cdot n s_{g} \cdot Y A_{a s g j}\right) \quad \forall s, g \in G_{s}, j \in J_{g}
$$

Constraints (7) calculate the total number of each type action $a$ to be performed by the agency.

$$
Y A T_{a}=\sum_{s} \sum_{g \in G_{s}} \sum_{j \in J_{g}} Y A_{\text {asgj }} \quad \forall a
$$

Constraints (8) and (9) establish the relationship between the number of stakeholders interested to participate in a project $\mathrm{p}$ of a certain call $\mathrm{j}$ and the binary variable indicating if some stakeholder of stage $\mathrm{s}$ participate in the corresponding project $\mathrm{p}$.

$$
\begin{array}{lr}
\sum_{g \in G_{s}} N_{\text {sgjp }} \leq M \cdot Y_{\text {sjp }} & \forall s, j, p \in P_{j} \\
Y_{s j p} \leq \sum_{g \in G_{s}} N_{s g j p} & \forall s, j, p \in P_{j}
\end{array}
$$

Constraints (10) and (11) ensure that a project proposal p should be presented only if there are stakeholders interested on it. Through constraints (12) the maximum number of project proposals that can be presented in a call is respected.

$$
Y_{s j p} \leq Y P_{j p} \quad \forall s, j, p \in P_{j}
$$




$$
\begin{array}{lr}
Y P_{j p} \leq \sum_{s} Y_{s j p} & \forall j, p \in P_{j} \\
\sum_{p \in P_{j}} Y P_{j p} \leq \operatorname{npmax}_{j} & \forall j
\end{array}
$$

It has no sense that stakeholders of the first stage participate in a project proposal if there are no stakeholders of the second stage. Because the laboratories participation only has sense if there are some technological stakeholder that need to do experimentation with the technology. Constraints (13) ensure this aspect.

$$
Y_{1 j p} \leq Y_{2 j p} \quad \forall j, p \in P_{j}
$$

Through constraints (14) it is not allowed to exceed the number of simultaneous projects that one stakeholder of a group is able to attend.

$$
\sum_{j} \sum_{p \in P_{j}} N_{s g j p} \cdot \delta_{j t} \leq n j_{g} \cdot n s_{g} \quad \forall s, g \in G_{s}, t
$$

At each time period, the consumed quantity of each resource by all the promoting actions implemented should not be greater than the available capacity of this resource in this time period (15).

$$
\sum_{a} \sum_{j} \operatorname{cons}_{r a j} \cdot \delta_{j t} \cdot Y A_{a j} \leq \operatorname{cap}_{r t} \quad \forall r, t
$$

The nature of the different decision variables is declared by means of the constraints (16).

$$
\begin{array}{ll}
N_{\text {sgjp }}, Y A T_{a} \geq 0, & \text { Integer } \\
Y_{s j p}, Y P_{j p}, Y A_{\text {asgj }} & \text { Binary }
\end{array}
$$

\section{Implementation and validation: application to a Development and Innovation Agency in Brittany}

The model was implemented in MPL $\AA 5.0 .8$ and solved by using the solver Gurobi ${ }^{\mathrm{TM}}$ 8.1.1 in a computer with an Intel ${ }^{\circledR}$ Xeon ${ }^{\circledR}$ CPU E5-1620 v2(C) @3.70GHz processor, with an installed capacity of $35 \mathrm{~GB}$ and a 64-bits operating system. Microsoft Access Database was used to store input data and results.

The above MILP model has been validated through a data set inspired in the case of a Development and Innovation Agency in Brittany that interact with stakeholders of the three stages indicated in Fig 1. It is assumed to be 15 groups of stakeholders: 5 groups in stage 1 with 10 members each one, 4 groups in stage 2 with 10 members 
each one, and 6 groups in stage 3 with 30 members each. The number of projects in which stakeholders can be simultaneously working are 3 for group 1, 5 for group 2 and 6 for group 3. It is assumed to be 7 project calls with a maximum of 4 projects to be presented per call. The open period for presenting each project call expressed in months can be seen in Fig 2 from which the $\delta_{j t}$ parameter can be defined. The minimum and maximum number of stakeholders for each stage and project call are: for stage 1, 0 and 7, for stage 2, 2 and 12 and for stage 3,15 and 40, respectively.

It is assumed the Agency has 2 limited resources: money and personnel time work. The agency can implement 5 types of actions: 1) send an e-mail with information about the project call, 2) to send a triptych, 3) to make a call phone, 4) to develop a stakeholder meeting days and 5) to visit the stakeholders. Each action type consumes a different quantity of both types of resources and has a certain impact as regards the stakeholders attracted to participate in different project call. They are defined in a way that the greater the impact of an action type the greater the resources consumption.

Time Horizon (months)

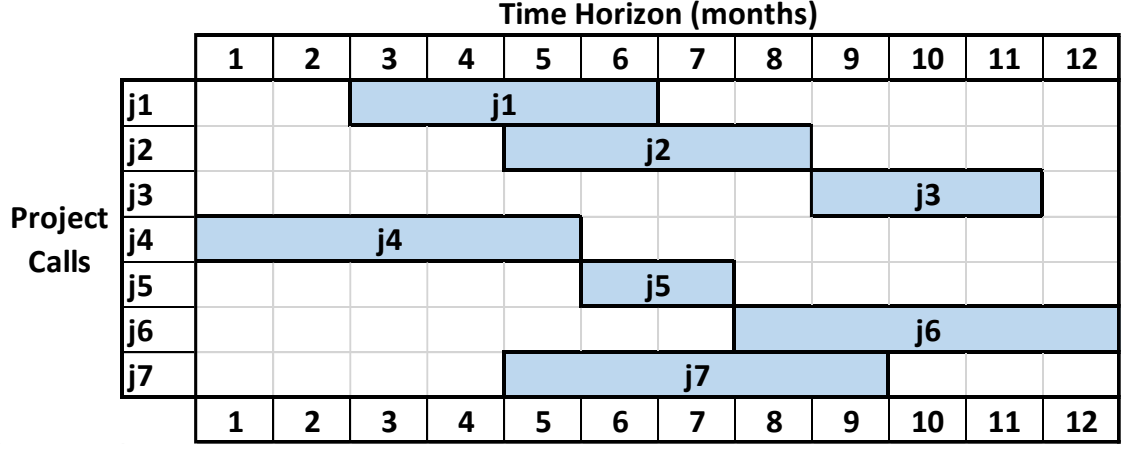

Fig. 2. Time period for presenting project proposals of each call (months).

In order to validate the MILP model proposed, we have assigned different weights to each objective function. This allows us to analyze the impact of these weights on the solutions obtained. In this case, only the money has been considered as the resource to be minimized (Z2) and only the personnel time work has been considered in constraints (14). Besides in order to analyze the model sensitivity to the impact of each action type $i a_{a g j}$, three scenarios have been defined (Table 4).

Table 4. Value of the action impact $\left(i a_{a g j}\right)$ per action type and scenario.

\begin{tabular}{|r|r|r|r|}
\hline Action Type & Base Scenario & Scenario 1 & Scenario 2 \\
\hline 1 & 0,025 & 0,030 & 0,020 \\
\hline 2 & 0,030 & 0,035 & 0,030 \\
\hline 3 & 0,040 & 0,045 & 0,100 \\
\hline 4 & 0,850 & 0,800 & 0,900 \\
\hline 5 & 0,600 & 0,400 & 0,300 \\
\hline
\end{tabular}


Results of the different weights combination as regards the two objective function can be consulted in (Fig. 3) for the three scenarios defined. As it can be observed in all three scenarios the number of projects presented and the investment diminish when the weight assigned to the $\mathrm{Z} 1$ decreases showing they are in conflict. It can be seen that to provide some weight to the Z2 $(w 2)$ inside the interval $[0.1,0.3]$ considerably decreases the amount of money invested by the Agency, while the number of projects presented practically remains the same. On the opposite, if the weight assigned to Z2 is higher than $0.6(w 2>=0.6)$ the number of projects presented considerably decreases being practically null in the interval $0.7<=w 2<=1$, as it was expected.

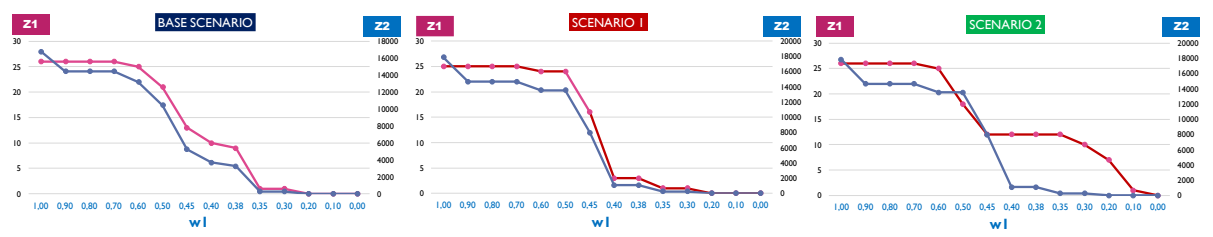

Fig. 3. Value of $Z 1$ and $Z 2$ for each scenario under different weights for the objective functions.

It is noteworthy that for each scenario there is a critical combination of weights around in the middle ( $\mathrm{w} 1=0.5$ and $\mathrm{w} 2=0.5$ ) where a little variation of weights originates high differences in the projects presented. Therefore, this aspect should be taken into account when defining the weights of each objective. From the different type of actions selected in each scenario, it can be deduced the importance of properly estimating the value of the impact of each action type $\left(i a_{a g j}\right)$.

Table 5. $\mathrm{N}^{\mathrm{o}}$ of each action type for different objective function weights and scenario

\begin{tabular}{|c|c|c|c|c|c|c|c|c|c|c|c|c|c|c|c|c|}
\hline & \multicolumn{5}{|c|}{ BASE SCENARIO } & \multicolumn{5}{|c|}{ SCENARIO 1} & \multicolumn{5}{|c|}{ SCENARIO 2} \\
\hline w1 & w2 & YAT(1) & YAT(2) & YAT(3) & YAT(4) & YAT(5) & YAT(1) & YAT(2) & YAT(3) & YAT(4) & YAT(5) & YAT(1) & YAT(2) & YAT(3) & YAT(4) & YAT(5) \\
\hline 1,00 & 0,00 & 43 & 29 & 47 & 5 & 18 & 42 & 32 & 46 & 14 & 6 & 9 & 7 & 51 & 17 & 5 \\
\hline 0,90 & 0,10 & 36 & 22 & 50 & 1 & 21 & 39 & 14 & 43 & 14 & 4 & 0 & 0 & 40 & 15 & 0 \\
\hline 0,80 & 0,20 & 36 & 22 & 50 & 1 & 21 & 39 & 14 & 43 & 14 & 4 & 0 & 0 & 40 & 15 & 0 \\
\hline 0,70 & 0,30 & 36 & 22 & 50 & 1 & 21 & 39 & 14 & 43 & 14 & 4 & 0 & 0 & 40 & 15 & 0 \\
\hline 0,60 & 0,40 & 31 & 13 & 40 & 1 & 21 & 20 & 0 & 28 & 14 & 6 & 0 & 0 & 57 & 11 & 3 \\
\hline 0,50 & 0,50 & 17 & 6 & 34 & 2 & 16 & 20 & 0 & 28 & 14 & 6 & 0 & 0 & 52 & 6 & 1 \\
\hline 0,45 & 0,55 & 16 & 0 & 21 & 0 & 10 & 10 & 0 & 14 & 8 & 4 & 4 & 4 & 60 & 1 & 0 \\
\hline 0,40 & 0,60 & 12 & 0 & 16 & 0 & 7 & 18 & 5 & 22 & 0 & 0 & 4 & 4 & 60 & 1 & 0 \\
\hline 0,38 & 0,62 & 21 & 3 & 21 & 0 & 5 & 18 & 5 & 22 & 0 & 0 & 4 & 4 & 60 & 1 & 0 \\
\hline 0,35 & 0,65 & 6 & 1 & 6 & 0 & 0 & 5 & 1 & 7 & 0 & 0 & 4 & 4 & 60 & 1 & 0 \\
\hline 0,30 & 0,70 & 6 & 1 & 6 & 0 & 0 & 5 & 1 & 7 & 0 & 0 & 0 & 0 & 52 & 1 & 0 \\
\hline 0,20 & 0,80 & 0 & 0 & 0 & 0 & 0 & 0 & 0 & 0 & 0 & 0 & 0 & 0 & 48 & 0 & 0 \\
\hline 0,10 & 0,90 & 0 & 0 & 0 & 0 & 0 & 0 & 0 & 0 & 0 & 0 & 0 & 0 & 5 & 0 & 0 \\
\hline 0,00 & 1,00 & 0 & 0 & 0 & 0 & 0 & 0 & 0 & 0 & 0 & 0 & 0 & 0 & 0 & 0 & 0 \\
\hline
\end{tabular}

\section{Conclusions and future research lines}

In this paper a decision support tool based on an ILP model has been developed to assist innovation agencies in deciding the type of actions to promote project proposals in the agricultural sector. The model has been validated through its application to a 
realistic case inspired on a Development and Innovation Agency in Brittany. From the results obtained it can be concluded that the two objectives considered in the model are in conflict. Therefore, it seems to be reasonable consider both them. Even more, for all scenarios, it is observed that assign even a low weight to the second objective can substantially diminish the money investment with a very small variation in the number of project proposals. Therefore, it is recommended to follow this practice. On the other hand, the results of the model are highly dependent on the impact of each action type, so this parameter should be defined as precisely as possible. Along these lines, future research lines are devised to include the modelling of uncertainty in some parameters such as the impact of action types.

Acknowledgments. The authors acknowledge the support of the project 691249, RUCAPS: "Enhancing and implementing knowledge based ICT solutions within high risk and uncertain conditions for agriculture production systems", funded by the European Union's research and innovation programme under the H2020 Marie Skłodowska-Curie Actions.

\section{References}

1. European Comission Funded Programs, https://ec.europa.eu/programmes/horizon2020

2. Zoie, C., Radulescu, M.: Decision Analysis for the Project Selection Problem Under Risk, IFAC Proceedings Volumes. 34 (8), 445--450 (2001)

3. Sadi-Nezhad, S.: A state-of-art survey on project selection using MCDM techniques. J. Project Manage. 2, 1--10 (2017)

4. Caballero, H.C., Chopra, S., Schmidt, E.K.: Project portfolio selection using mathematical programming and optimization methods. Paper presented at PMI ${ }^{\circledR}$ Global Congress 2012 North America, Vancouver, British Columbia, Canada. Newtown Square, PA: Project Management Institute. (2012)

5. Ahmad, B., Haq, I.: Project Selection Techniques, Relevance \& Applications in Pakistan. Int. J. Technol. Res. 4, 52--60 (2016)

6. Inuiguchi, M., Ramík, J.: Possibilistic linear programming: a brief review of fuzzy mathematical programming and a comparison with stochastic programming in portfolio selection problem, Fuzzy Sets Syst. 111(1), 3--28 (2000)

7. Stewart, R., Mohamed, S.: IT/IS projects selection using multi-criteria utility theory, Log. Inf. Manage. 15(4), 254--270 (2002)

8. Alzober, W., Yaakub, A.R.: Integrated model for MCDM: Selection contractor in Malaysian construction industry. In Applied Mechanics and Materials 548, pp.1587--1595). Trans Tech Publications. (2014)

9. Adhikary, P., Roy, P.K., Mazumdar, A.: Optimal renewable energy project selection: A multi-criteria optimization technique approach. Global J. Pure Appl. Math. 11(5), 3319--3329 (2015)

10. Strang, K.D.: Portfolio selection methodology for a nuclear project. Project Manage. J. 42(2), 81--93 (2011)

11. Benjamin, C.O.: A linear goal-programming model for public-sector project selection. J. Oper. Res. Soc. 13--23. (1985)

12. Coronado, J.R., Pardo-Mora, E.M., Valero, M.: A Multi-objective model for selection of projects to finance new enterprise SMEs in Colombia. J. Ind. Eng. Manage. 4(3), 407--417 (2011) 
13. Mat, N.A.C., Cheung, Y.: Partner selection: criteria for successful collaborative network. In: 20th Australian Conference on Information Systems, 631--641 (2009)

14. Camarinha-Matos L.M., Afsarmanesh H.: Collaborative Networks. Value creation in a knowledge society in International Federation for Information Processing (IFIP), Volume 207, Knowledge Enterprise: Intelligent Strategies In K. Wang, Kovacs G., Wozny M., Fang M. (eds), 26-40. Springer, Boston (2006).

15. Paixão, M., Sbragia, R., Kruglianskas, I.: Factors for selecting partners in innovation projects - evidences from alliances in the brazilian petrochemical leader. Rev. Admin. Innov. São Paulo, 11(2), 241--272 (2014)

16. Duisters, D., Duysters, G., de Man, A.P.: The partner selection process: Steps, effectiveness, governance. Ann. Hematol. 2, 7--25 (2011)

17. Zhang, X.: Criteria for Selecting the Private-Sector Partner in Public-Private Partnerships. J. Constr. Eng. Manage. 131(6), 631--644 (2005) 